\title{
Hierarchical petascale simulation framework for stress corrosion cracking
}

Article in Journal of Physics Conference Series · August 2007

DOI: $10.1088 / 1742-6596 / 78 / 1 / 012036$

CITATIONS

2

11 authors, including:

\section{Aiichiro Nakano}

University of Southern California

357 PUBLICATIONS $\quad 6,027$ CITATIONS

SEE PROFILE

\section{Gang Lu}

California State University, Northridge

141 PUBLICATIONS 2,253 CITATIONS

SEE PROFILE
Efthimios Kaxiras

Harvard University

462 PUBLICATIONS 18,388 CITATIONS

SEE PROFILE

Stephan Eidenbenz

Los Alamos National Laboratory

139 PUBLICATIONS 2,217 CITATIONS

SEE PROFILE

Some of the authors of this publication are also working on these related projects:

Project Electronic and magnetic properties of spintronic materials View project 


\title{
Hierarchical Petascale Simulation Framework for Stress Corrosion Cracking
}

\author{
P. Vashishta (PI), R. K. Kalia and A. Nakano (University of Souther California) \\ E. Kaxiras (Harvard Univ.), A. Grama (Purdue Univ.), G. Lu (California State-Northridge) \\ S. Eidenbenz and A. F. Voter (Los Alamos Nat'l Laboratory) \\ R. Q. Hood, J. A. Moriarty and L. H. Yang (Lawrence Livermore Nat'l Laboratory)
}

The goal of this SciDAC project is to develop a scalable parallel and distributed computational framework consisting of methods, algorithms, and integrated software tools for: 1) multi Tera-toPetascale simulations with quantum-level accuracy; 2) multimillion-to-multibillion-to-trillion atom molecular dynamics (MD) simulations based on density functional theory (DFT) and temperature dependent model generalized pseudopotential theory; 3) quasicontinuum (QC) method embedded with classical atomistic and quantum simulations based on DFT; and 4) accelerated molecular dynamics (AMD) coupled with hierarchical atomistic/QC simulations to reach macroscopic length and time scales relevant to SCC. Scalability is being achieved beyond $10^{5}$ processors through linear-scaling algorithms and performance-optimization techniques. We are employing automated model transitioning to embed higher fidelity simulations concurrently inside coarser simulations only when and where they are required. Each scale and model has well-defined error bounds with controlled error propagation across the scales and models to estimate uncertainty in predictions.

\section{RESEARCH ACCOMPLISHMENTS}

\section{Billion-atom MD Simulation of Nanoscale Ductile Damage in Silica Glass}

Cavitation is a ubiquitous form of damage in the ductile fracture of metallic alloys $^{2}$. Voids in metallic alloys nucleate at grain-boundary junctions and from decohesion or cracking of inclusions $\mathrm{s}^{3,4}$. With an increase in the applied load, voids grow and coalesce to mediate ductile failure ${ }^{5}$.

A long-held belief is that brittle materials fracture solely by de-cohesion of atomic bonds at the crack tip ${ }^{16}$. However, recent Atomic Force Microscopy (AFM) studies have generated considerable controversy about the nature of damage in stress corrosion cracking of brittle amorphous solids. The Saclay group observes the nucleation, growth and coalescence of nanometer scale cavities as the key mechanism for crack extension in silica and aluminosilicate glasses ${ }^{17}, 18$, whereas the NIST group finds no experimental evidence for cavitation around a crack in silica glass 19 .

We have performed multimillion-to-billion atom MD simulations to investigate dynamic mechanisms of void coalescence and fracture in silica glass under hydrostatic tension. First, we validated the interatomic potential used in our simulation with first-principles density functional calculations using a plane-wave pseudopotential method [1] within the density functional theory approach [2] in conjunction with a parameter independent generalized gradient approximation [3] for the exchange-correlation interactions. A 192-atom amorphous silica system prepared by an MD simulation was fully relaxed by the DFT method. The excellent agreement in the network topology of a- $\mathrm{SiO}_{2}$ between DFT and classical MD simulations reveals the high quality of the classical interatomic potential for $\mathrm{SiO}_{2}$. More importantly, the forces on $\mathrm{Si}$ and $\mathrm{O}$ atoms calculated from DFT and MD simulation with classical interatomic potential for the same a- $\mathrm{SiO}_{2}$ configuration deviate less than $4.5 \%$ and $6 \%$, respectively.

Figure 1 shows evolution and coalescence among multiple voids in a billion-atom system subjected to a dilatational strain. The initial configuration is an array of 500 voids, the initial diameter of each void is $3 \mathrm{~nm}$, and the nearest void center-tocenter separation is $6 \mathrm{~nm}$. Our simulations show that void-void interactions in amorphous silica ensue with the formation of nanometer scale cavities in inter-void ligaments and are soon followed by the growth of cracks on void surfaces and

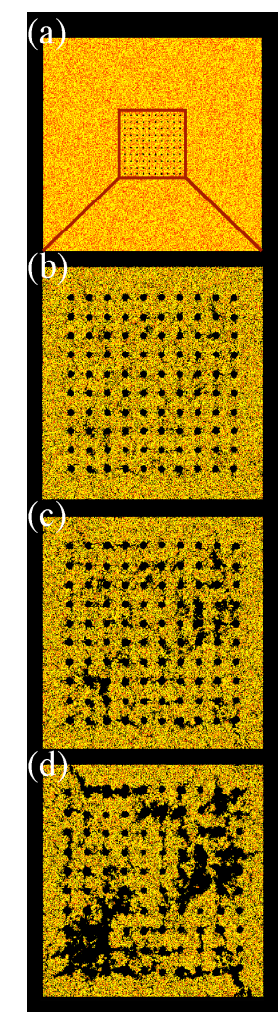

Fig. 1: Snapshots of voids in a 1-billion atom a- $\mathrm{SiO}_{2}$ system under hydrostatic strain. (b), (c) and (d) show void growth, cracks on void surfaces, and inter-void ligament failure. 
failure of ligaments due to the growth and coalescence of voids through ligament cavities. Many features of void-void interaction and coalescence in silica glass are surprisingly akin to those observed in ductile metallic alloys.

Detailed analysis of cavity nucleation reveals a novel mechanism involving strain-enhanced defect transport; see Fig. 2. Here red and yellow are silicon and oxygen atoms, respectively. Each $\mathrm{Si}$ atom is connected to $4 \mathrm{O}$ atoms in the form of a tetrahedron and the $\mathrm{SiO}_{4}$ tetrahedra are linked into nanometer size rings through corner-sharing $\mathrm{O}$ atoms. These rings expand and distort under strain to form nanometric cavities. In Fig. 2(a), blue, green and gray regions are inside 7membered, 6-membered and 5-membered $\mathrm{Si}-\mathrm{O}$ rings, respectively. The blue atom in Fig. 2(a) is an under-coordinated $\mathrm{O}$ bonded to a $\mathrm{Si}$ in the 7membered blue ring. The oxygen atoms, shown in green and white, play a pivotal role in the nucleation of a nanocavity. Figure 2(b) shows that at a strain of $1 \%$ the under-coordinated $\mathrm{O}$ (blue) becomes fully coordinated by binding with a $\mathrm{Si}$ while the white $\mathrm{O}$ atom becomes under-coordinated by dissociating from that $\mathrm{Si}$ atom. As a result, the 5membered gray ring becomes a 12 -membered ring and the blue oxygen is now a part of a 3-membered ring. At a strain of $4 \%$, the green $\mathrm{O}$ dissociates from a $\mathrm{Si}$ atom and the latter binds with the white $\mathrm{O}$ atom, thus creating an 11-membered ring (gray) adjacent to a 10-membered ring (green); see Fig. 2(c). The transport of $\mathrm{O}$ defects is driven by stress gradients. This mechanism introduces a natural length scale, i.e., ring diameter $(\sim 1 \mathrm{~nm})$, into the nanocavity nucleation problem.

\section{HYBRID QMD-MGPT/MD ALGORITHM FOR OPTIMIZED Potentials}

Bridging the length and time scale gaps from quantum mechanics to large scale atomistic simulation is a key required element in the firstprinciples multiscale modeling of SCC. Modeling across the quantumatomistic gap has become possible in recent years through the development of quantum-based interatomic potentials derived from DFT-based generalized pseudopotential theory (GPT). For complex transition metals of interest in SCC, including $\mathrm{Ti}$ and $\mathrm{Ni}$ alloys, the simplified model GPT or MGPT method is a useful practical approach for developing accurate many-body potentials for large scale simulations $[1,2]$. Heretofore, however, MGPT potentials have been based on zerotemperature electrons, a treatment that neglects important electronthermal contributions to the high-temperature solid and to the melt in transition metals. In addition, quantum simulations in metals, such as quantum molecular dynamics (QMD), naturally include electron temperature and provide a much richer DFT data set for accurate potential development. We are developing a hybrid QMD-MGPT/MD simulation capability for transition metals and alloys that will allow us to harvest optimized and quantum-level-accurate temperature-dependent MGPT potentials for SCC applications.

Our hybrid QMD-MGPT/MD method is illustrated schematically in Fig. 3. In this scheme alternate QMD and MGPT/MD simulation segments are linked together in a single code with information flow between the
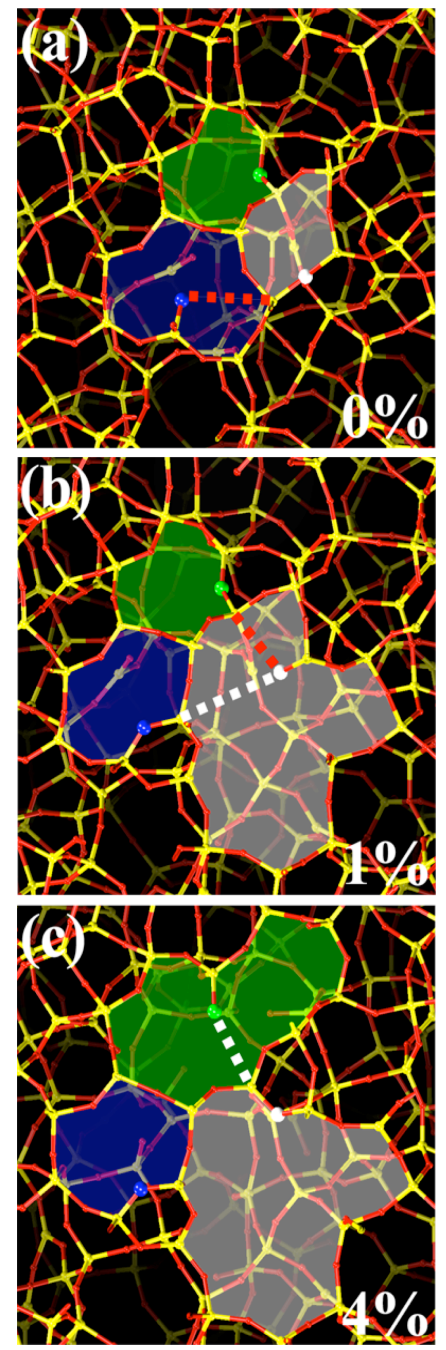

Fig. 2: Atomic configurations of bond switching mechanisms. Red and yellow spheres represent $\mathrm{Si}$ and $\mathrm{O}$ atoms, respectively. Dashed lines indicate the locations of bond formation and rupture during bond switching, which involves two oxygen atoms. The bond switching between blue and white oxygens in Figs. 2(a) and 2(b) is shown by red and white dashed lines. In Fig. 2(c), the white dashed line indicates bond switching between white and green oxygen atoms.

segments. In particular, both snapshot and accumulated QMD data can be passed forward to update the MGPT potentials on the fly in an iterative fashion. Working on small systems suitable for QMD, we thereby expect to obtain optimized MGPT potentials in the long time limit of the hybrid simulation, potentials which can then be re-used in petascale atomistic simulations for SCC. The QMD segments of 
the hybrid simulation are directly implemented with our parallel plane-wave pseuodpotential DFT code $\mathrm{P}^{3} \mathrm{MD}$. This code has been modified to accommodate MGPT forces and the MGPT/MD segments of the hybrid simulation. We are currently working on an information passing strategy to optimize the MGPT potentials, beginning with a Mo prototype and existing zero-temperature, pressure-dependent potentials. In this regard, we have discovered a useful scaling parameter such that a smooth transition, as measured by the temperature fluctuation from a QMD to an MGPT/MD segment of the simulation, can be achieved after a few iterations.

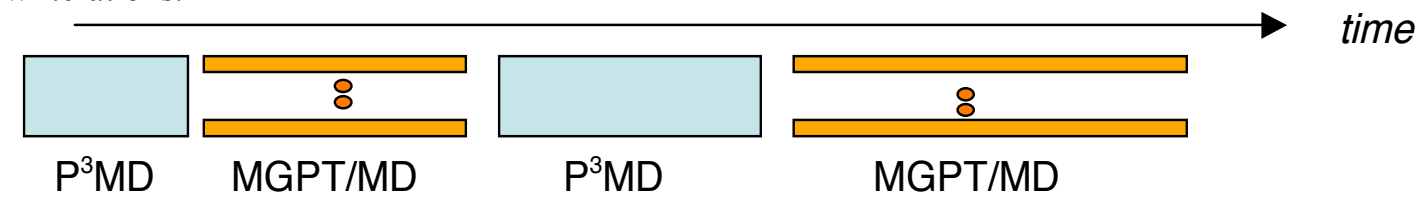

Fig. 3: Schematic representation of the hybrid QMD-MGPT/MD algorithm. A real-time information feedback loop between the alternate QMD ( $\left.\mathrm{P}^{3} \mathrm{MD}\right)$ and MD/MGPT segments of the simulation will allow optimized MGPT potentials to be obtained in the long time limit.

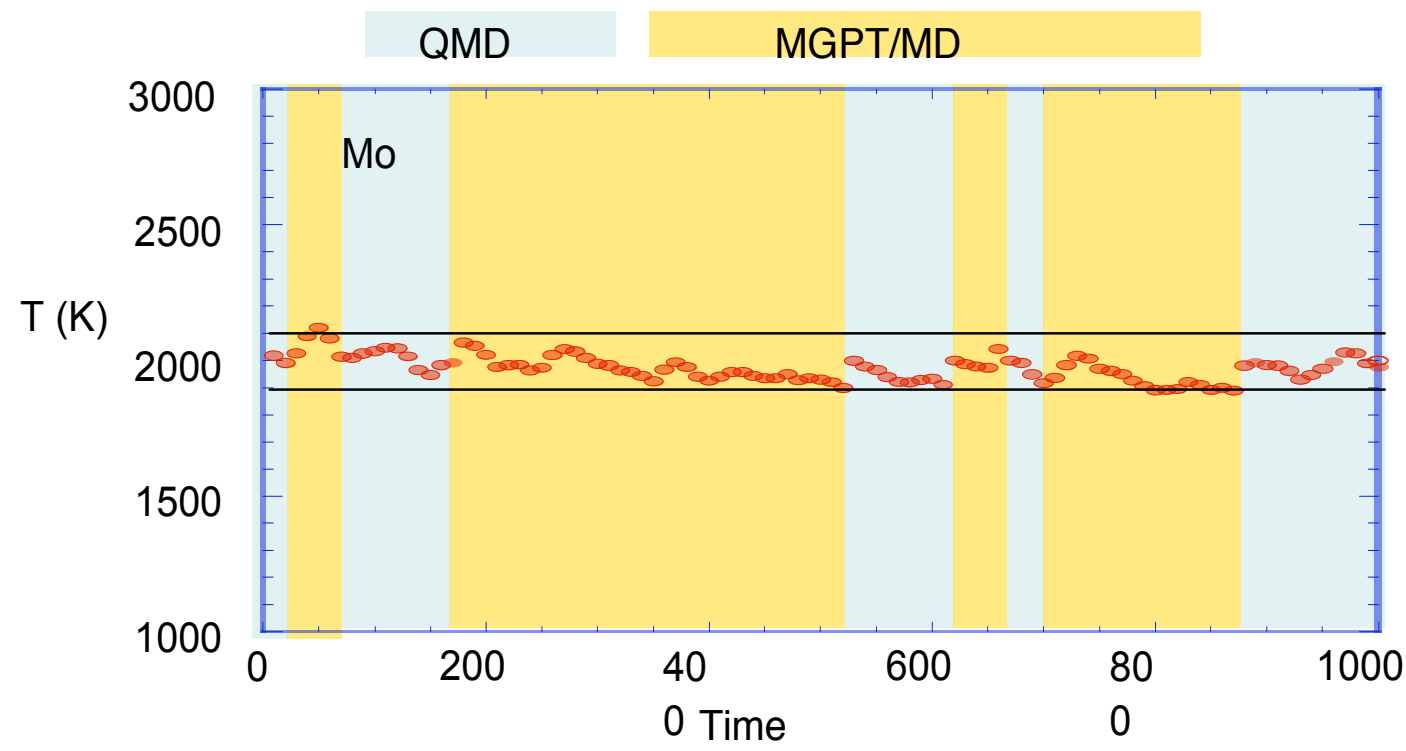

(fs)

Fig. 4: The temperature fluctuation of 250-atom Mo system simulated with our hybrid QMD-MGPT/MD algorithm.

Our initial constant-temperature hybrid simulation results for Mo are shown in Fig. 4. Here we started with a QMD segment for 250 Mo atoms at $\mathrm{T}=0 \mathrm{~K}$ and gradually heated the system up to $2000 \mathrm{~K}$ over about 50 time steps. At the end of the QMD segment, we took the average positions and velocities of the last five time steps as the input information for the initial MGPT/MD segment. A sudden jump of the temperature by $15 \%$ was observed when the first MGPT/MD simulation segment was started. We then allowed the MGPT/MD simulation to continue until the average temperature reached $2000 \mathrm{~K}$, which we used as the condition to start the second QMD segment with input derived from the average positions and velocities of the last five time steps from the MGPT/MD segment. The second segment of the QMD simulation was performed for a longer

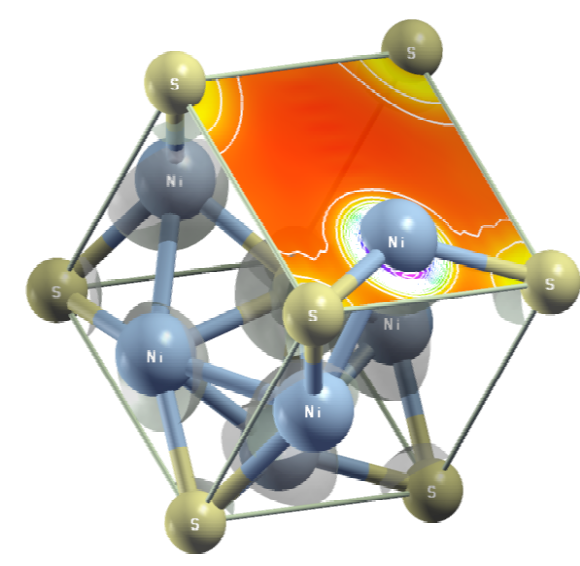

Fig. 5: Electronic structure of idealized heazlewoodite $\mathrm{Ni}_{3} \mathrm{~S}_{2}$ system. 
time of about $10 \mathrm{ps}$, which was sufficient to reach the equilibrium temperature of $2000 \mathrm{~K}$. The statistics accumulated from the three segments (2 QMD and one MGPT/MD segment) suggested that our timedependent scaling parameter be defined as $\alpha=\left(<T_{\mathrm{MGPT}}>/<T_{\mathrm{QMD}}>\right)^{1 / 2}$. The initial value of $\alpha$ was found around to be 1.30 and this was gradually reduced to 1.02 over the next three transitions between QMD and MGPT/MD segments of the simulation.

In addition, we have calculated the electronic properties of this system. In particular, the density of the states at the Fermi level $\mathrm{N}\left(\mathrm{E}_{\mathrm{F}}\right)=2.352$ states/eV-cell. This leads to a band-structure electronic-heatcapacity constant of $\gamma=5.559 \mathrm{~mJ} / \mathrm{mol}^{-} \mathrm{K}^{2}$, where $1 \mathrm{~mol}$ refers to $1 \mathrm{~mol}$ of $\mathrm{Ni}_{3} \mathrm{~S}_{2}$. This value can be compared with the experimental value [3] of $\gamma_{\exp }=8.1 \mathrm{~mJ} / \mathrm{mol}-\mathrm{K}^{2}$. This gives a electron-phonon enhancement factor of $\lambda_{\mathrm{e}-\mathrm{p}}=\gamma_{\mathrm{exp}} / \gamma-1=0.457$, indicating that the electron-phonon coupling is moderate and therefore the temperature-dependent MPGT potentials are necessary for studying SCC problems in this system.

We have also performed a first-principles electronic structure calculation of an idealized $\mathrm{Ni}_{3} \mathrm{~S}_{2}$ system illustrated in Fig. 5. The bulk properties of this system are important material parameters that will be used to develop MGPT interatomic potentials for Ni-S system. The calculated lattice constant for the ideal structure is $4.071 \mathrm{~A}$ and three cubic elastic constants are $\mathrm{C}_{11}=198 \mathrm{GPa}, \mathrm{C}_{12}=113 \mathrm{GPa}$, and $\mathrm{C}_{44}=108$ $\mathrm{GPa}$.

\section{Multiscale Modeling of Metallic Systems: From Electrons to Finite- ELEMENTS}

We have recently developed three distinctive $\mathrm{QM} / \mathrm{MM}$ multiscale approaches that couple quantum mechanical $(\mathrm{QM})$ calculations to classical atomistic simulations $(\mathrm{MM})$ for metals. The first approach deals with the coupling between Kohn-Sham density-functional theory (KS-DFT) and EAM classical atomistic method with the coupling energy calculated classically by EAM. The second approach involves coupling between orbital-free density functional theory (OFDFT) and EAM but with the coupling energy calculated quantum mechanically by OFDFT. The third approach is similar to the first one, but with the interaction energy calculated quantum mechanically by OFDFT. Unlike most of the QM/MM approaches, our methods (the last two) allow charge transfer between QM and MM regions. The electrons in the QM region feel not only the Kohn-Sham effective potential, but also an additional embedding potential generated by electrons (Hartree, exchange and correlation and kinetic energy contributions) and ions (pseudopotential contribution) in the MM region. This embedding potential is evaluated rigorously by OFDFT. The first approach has been incorporated into the Quasicontinuum $(\mathrm{QC})$ method, which is a multiscale approach that seamlessly combines classical atomistic calculation with finite-element simulations. We have thus achieved one of the goals of this SciDAC project, namely, the development of concurrent multiscale approaches (QCDFT) that span length scales from electrons to finite-elements for metallic systems. Currently, we are incorporating the second and third coupling approaches into the QC method.

We have applied the multiscale methods to study crack propagation in $\mathrm{Al}$ in the presence/absence of hydrogen impurities at the crack tip (Fig. 6). We find partial dislocations are nucleated at the crack tip and they move away from the tip under stress. These partial dislocations blunt the crack tip and cause ductile fracture in the absence of $\mathrm{H}$ impurities. When $\mathrm{H}$ impurities are introduced, the fracture toughness is significantly reduced.

We have also performed multiscale simulations for homogenous nucleation of vacancy clusters in fcc metals (including $\mathrm{Al}, \mathrm{Cu}, \mathrm{Ni}, \mathrm{Pd}, \mathrm{Ag}, \mathrm{Au}, \mathrm{Pt}$ and Ir). We computed Gibbs free binding energy of vacancy clusters in $\mathrm{Al}$ and carried out thermodynamic analysis of void formation. We introduced a critical nucleus size for void formation and examined the dependence of the critical size on temperature and hydrostatic pressure. We found that while pressures can enhance void formation, tensions tend to inhibit or prohibit it. We discovered that the "bonding cages" (Fig. 7) play a crucial role in void formation in fcc materials. The formation of the "bonding cages" significantly reduces the propensity of void formation. A simple model is proposed to explain the connection of "bonding cage" and the tendency of void formation for all fcc metals. Finally, we find that the formation of "bonding cage" is closely related to the directionality of the bonding in the metals. The two aforementioned applications are relevant to stress corrosion cracking (SCC) because they represent two major modes of failure observed in SCC. 


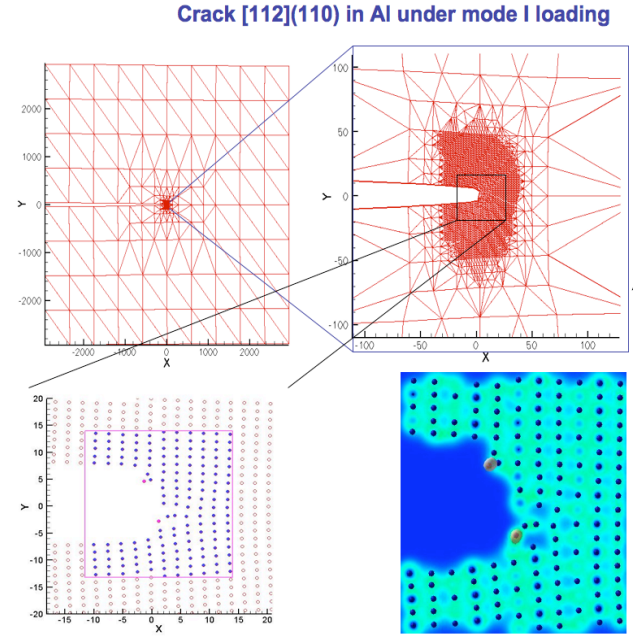

Fig. 6: Crack propagation with/without $\mathrm{H}$.
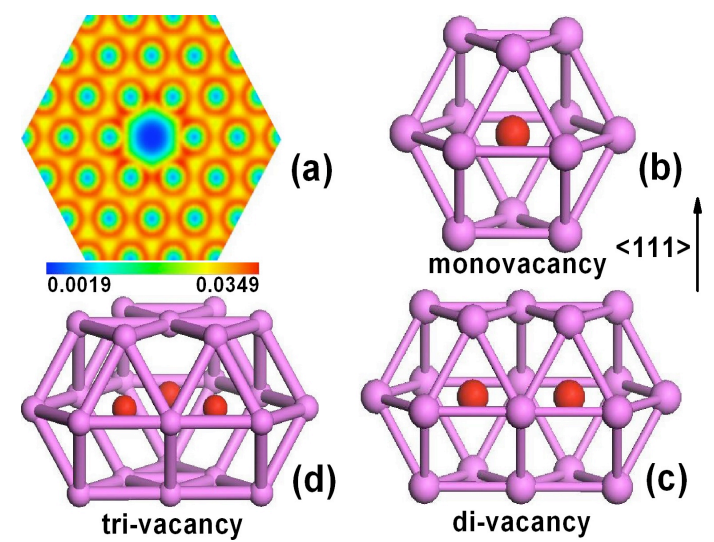

Fig. 7: Role of bonding cages in void formation in fcc metals.

\section{ACCELERATED DYNAMICS FOR LONG-TIME BEHAVIOR}

As discussed above, accurate simulation of stress-corrosion cracking depends in an essential way on building a hierarchical multiscale framework. At the largest size scale, the externally applied stress must be properly imposed on the system, requiring a continuum-level (e.g., finite-element or quasicontinuum) treatment. This information is passed to successively finer, more accurately described, levels. Ultimately, at the finest level, a small number of the most crucial atoms at the crack tip (region A, order $10^{2}$ atoms) are modeled with a full electronic structure calculation (e.g., DFT). To reach appropriate time scales, accelerated molecular dynamics (AMD) simulations are employed on a small region (region $\mathrm{B}, \sim 10^{3}$ atoms) that includes the DFT region A as a subset. The atoms in region B other than the DFT atoms are described with a highly accurate semiempirical potential. After the entire system has been brought into thermal equilibrium using molecular dynamics coupled to finite element method (FEM), a shell of atoms outside region $\mathrm{B}$ are held fixed at their time-averaged positions to provide proper boundary conditions for region $\mathrm{B}$ while an AMD simulation is performed within that region. This AMD simulation proceeds until a thermally activated transition takes this subsystem to a new state. For example, this may consist of a chemical reaction at the crack tip that allows the crack to advance by one lattice constant. At this point, communication with the rest of the system is required, to allow the strain field of the overall system to re equilibrate with the modified region B. The newly relaxed shell of atoms around region B is then fixed in place again and new AMD is performed until the next reactive event. This procedure is repeated to advance the infrequent-event dynamics of the entire system. In a more complex case, such as if a dislocation is emitted from the crack tip and passes from region $\mathrm{B}$ into region $\mathrm{C}$, additional steps are required for proper treatment, but we won't consider such a case here.

A key aspect of this problem is that even though region A is reduced to the smallest possible size, the DFT calculations still dominate the computational work for the entire system. Moreover, this computational expense is so great that it even threatens to prevent the use of the AMD methods to reach longer time scales. This is because a certain trajectory time must be invested as overhead work before boost in the time scale starts to be achieved. For the parallel-replica dynamics (PRD) method [V1], in which time is parallelized, this overhead corresponds to the dephasing time (typically a few ps) during which the individual replicas are made independent of each other. Thus, if the DFT calculations are so expensive that the computationally feasible total trajectory time is only a few ps, then AMD is not helpful, even on a petaflop machine, and the total time the stress-corrosion cracking system can be advanced will be a few ps, using direct MD on a subset of the processors.

We have recently discovered a way to circumvent this potentially show-stopping problem with a new approach for treating the dephasing time. In region A, rather than always using the full DFT treatment, we will instead use our fast semi-empirical potential during the dephasing stage. This introduces a new 
approximation, as the inaccuracy of the semi-empirical potential means that the distribution of dephased replicas will not be sampled from the correct Boltzmann distribution for the DFT energy, and we are currently analyzing the nature and impact of this error by integrating the Klein-Kramers equation for model systems. If necessary, the distribution can be improved by running an additional short segment of dephasing trajectory using full DFT, or by mixing in a small amount of DFT during the dephasing with either a learn-on-the-fly approach [V2] or the method recently proposed by Taylor et al [V3]. However, it is noteworthy that in the limit where no DFT is required during dephasing, so that the dephasing cost is essentially zero, this new approach offers the possibility of nearly complete parallel scaling for the time acceleration. This thus represents a breakthrough, as the full power of the petaflop architecture can now be brought to bear on the stress-corrosion cracking problem. Moreover, because this overhead issue has in general stood in the way of using AMD methods with DFT forces, this new approach will have a broad impact beyond this SCIDAC project.

\section{Petascale Simulation Framework for Stress Corrosion Cracking}

The following are some of the foreseen challenges and proposed solutions that the simulation framework will guarantee:

\begin{tabular}{|c|c|}
\hline Challenges & \begin{tabular}{|c|} 
Proposed Solution \\
\end{tabular} \\
\hline $\begin{array}{c}\text { How to automatically turn on and off the } \\
\text { simulation packages }\end{array}$ & $\begin{array}{l}\text { Integrate the different simulation packages in the } \\
\text { software framework, such that they can be automatically } \\
\text { turned on and off back and forth without any external } \\
\text { control after the simulation starts. }\end{array}$ \\
\hline Framework Architecture & $\begin{array}{c}\text { The simulation framework will facilitate a plug-in } \\
\text { module architecture, wherein different simulation } \\
\text { modules can be plugged in or out at will to fit different } \\
\text { problem scenarios. }\end{array}$ \\
\hline High-Performance Computing & $\begin{array}{l}\text { The simulation packages will be run on peta-flop } \\
\text { machines. We will ensure that application launches } \\
\text { smoothly and is scalable. }\end{array}$ \\
\hline Understanding the crack-tip propagation & $\begin{array}{l}\text { - Re-assigning different sets of atoms in different } \\
\text { regions and coupling them together in the entire } \\
\text { simulation time-line. } \\
\text { - Autonomous data analysis and information } \\
\text { extraction and learning between the different } \\
\text { stages of the simulations. } \\
\text { - Software framework will ensure }\end{array}$ \\
\hline $\begin{array}{c}\text { Language Compatibility between simulation } \\
\text { packages }\end{array}$ & $\begin{array}{l}\text { The software framework will write a wrapper around } \\
\text { each package, so the different packages exchange } \\
\text { information gracefully. }\end{array}$ \\
\hline Resource Discovery and Scheduling & $\begin{array}{l}\text { The software framework will ensure resource discovery } \\
\text { and scheduling for the simulation packages that need } \\
\text { parallelism. }\end{array}$ \\
\hline Cluster Communication & $\begin{array}{l}\text { The software framework will ensure coherent exchange } \\
\text { of information between different nodes in a cluster and } \\
\text { exchanging the right data among the right nodes }\end{array}$ \\
\hline Simulation Parameters & $\begin{array}{l}\text { Simulation parameters will be specified through } \\
\text { scripting files or config files. }\end{array}$ \\
\hline Intra-package Communication & $\begin{array}{l}\text { Different parts of simulation package can run on } \\
\text { different machines. In such a scenario, we plan to use } \\
\text { remote procedure calls (RPC) between the different } \\
\text { interacting parts of the simulation packages }\end{array}$ \\
\hline
\end{tabular}




\section{References}

[1] Quantum-Based Atomistic Simulation of Materials Properties in Transition Metals, J. A. Moriarty, J. F. Belak, R. E. Rudd, P. Söderlind, F. H. Streitz, and L. H. Yang, J. Phys.: Condens. Matter 14, 2825 (2002).

[2] Robust Quantum-Based Interatomic Potentials for Multiscale Modeling in Transition Metals, J. A. Moriarty, L. X. Benedict, J. N. Glosli, R. Q. Hood, D. A. Orlikowski, M. V. Patel, P. Söderlind, F. H. Streitz, M. Tang and L. H. Yang, J. Mater. Research 21, 563 (2006).

[3] P. A. Metcalf, B. C. Crooker, M. McElfresh, Z. Ka,kol, and J. M. Honig, Phys. Rev. B 50, 2055 (1994).

[4] Y. Liu, G. Lu, Z. Chen and N. Kioussis, "An improved QM/MM approach for metals", Model. Simul. Mater. Sci. Eng. 15, 275 (2007).

[5] X. Zhang and G. Lu, "Homogeneous nucleation of vacancy cluster in f.c.c. metals", submitted.

[V1] A.F. Voter, Phys. Rev. B57, 13985 (1998).

[V2 ] G. Csanyi, T. Albaret, M. C. Payne, and A. DeVita, Phys. Rev. Lett. 93, 175503 (2004).

[V3] D.E. Taylor, V.V. Karasiev, K. Runge, S.B. Trickey, and F.E. Harris, Computational Materials Science 39, 705 (2007). 